\title{
HARMONIC ANALYSIS ON INTERNALLY GELFAND PAIRS ASSOCIATED TO GROUPOIDS
}

\section{IBRAHIMA TOURE*, KINVI KANGNI}

\author{
UFR de Mathématiques et Informatique
}

Université Félix Houphouet-Boigny; 22 BP 582 Abidjan 22, COTE-D'IVOIRE

${ }^{*}$ Corresponding author: ibrahima.toure@univ-ufhb.edu.ci; toure@aims.ac.za

\begin{abstract}
Let $G$ be a topological locally compact, Hausdorff and second countable groupoid with a Haar system and $K$ a proper subgroupoid of $G$ with a Haar system too. $(G, K)$ is an internally Gelfand pair if for any $u$ in the unit space, the algebra of bi- $K(u)$-invariant functions on $G(u)$ is commutative under convolution. In this work, we give some characterizations of these pairs and extend to this context some classical results of harmonic analysis.
\end{abstract}

\section{INTRODUCTION}

The notion of Gelfand pair, introduced by I.M.Gelfand, has been extensively studied on groups in papers such as $[1,4,5,7-10]$. It has permitted to extend many results of commutative harmonic analysis to noncommutative case. The notion of groupoid is an extension of the notion of group. In [21,22], we have extended the notion of Gelfand pair from groups to groupoids. In these papers, our analysis is done on a transitive locally compact groupoid, $G$, and a compact subgroupoid, $K$. For instance, in [21] we have proved that $(G, K)$ is a Gelfand pair if and only if for any $u \in G^{(0)}$ the pair of isotropy groups $(G(u), K(u))$ is a Gelfand pair in group sense. Thanks to this result, we have extended some results of harmonic analysis from groups to groupoids. But this result is not true in general. For instance, the groupoid algebra is

Received 2018-11-29; accepted 2019-01-30; published 2019-11-01.

2010 Mathematics Subject Classification. 22A22, 46J10.

Key words and phrases. groupoids; groupoid representation; internally Gelfand pairs.

(C)2019 Authors retain the copyrights of their papers, and all open access articles are distributed under the terms of the Creative Commons Attribution License. 
not necessarily commutative even for abelian groupoids that means groupoids with abelian isotropy groups. Nonetheless, there is a "nice" harmonic analysis on abelian groupoids $[3,11,16]$. So our purpose is, in order to do harmonic analysis on pairs $(G, K)$ for general locally compact groupoids, to define an alternative notion of Gelfand pairs on groupoids taking into account only the isotropy groups. Since any compact groupoid is proper and if $K$ is proper then the isotropy groups $K(u)$ are compact, we have giving our definition for proper subgroupoid, $K$. In fact, for a locally compact groupoid $G$ and a proper subgroupoid $K,(G, K)$ is an internally Gelfand pair if for any $u$ in the unit space, the algebra of bi- $K(u)$-invariant functions on $G(u)$ is commutative under convolution. After notations and setup in the next section, we give in section 3 some characterizations of internally Gelfand pairs, in particular we show that a Gelfand pair is an internally Gelfand pairs. We also study the link between Gelfand pairs and internally Gelfand pairs. In section 4 , we define the notion of $G^{(0)}$-spherical function associated to internally Gelfand pairs and study some properties of these functions. We establish a connection between $G^{(0)}$-spherical functions and internally irreducible representations introduce by R. Bos in his paper [3]. In section 5, we give an extension of Bochner Theorem.

\section{PRELIMINARIES}

We use the notations and setup of this section in the rest of the paper without mentioning. For basic notions on groupoids and Haar systems, we refer the reader to [20]. Throughout $G$ will be a second countable locally compact Hausdorff groupoid with unit space $G^{(0)}$ and left Haar system $\left\{\lambda^{u}, u \in G^{(0)}\right\}$. $G^{(2)}$ will denote the set of composable pairs. For $x \in G, r(x)=x x^{-1}$ and $d(x)=x^{-1} x$ are respectively the range and the domain of $x$. For $u, v \in G^{(0)}$, let us put $G^{u}=r^{-1}(u), G_{v}=d^{-1}(v), G_{v}^{u}=G^{u} \cap G_{v}$ and for each unit element $u, G(u)=\{x \in G: r(x)=d(x)=u\}$ is the isotropy group at $u$. The set $G^{\prime}=\{x \in G: r(x)=d(x)\}$ is the isotropy group bundle of $G$. The relation on $G^{(0)}$ defined by: $u, v \in G^{(0)}, u \sim v$ iff $G_{v}^{u} \neq \emptyset$ is an equivalence relation. The equivalence class of $u$ is denoted by $[u]_{G}$ and is called the orbit of $u$. The graph $R=\{(r(x), d(x)): x \in G\}$ of this equivalence relation is a groupoid with unit space $G^{(0)}$. The anchor map $\theta=(r, d)$ is a continuous homomorphism of $G$ into $G^{(0)} \times G^{(0)}$ with image $R$. A groupoid is transitive if $\theta$ is onto i.e. the range of $\theta$ is equal to $G^{(0)} \times G^{(0)}$. Otherwise, a groupoid is transitive if it has a single orbit. A groupoid is proper if $\theta$ is a proper map. For $u \in G^{(0)}, \lambda_{u}$ will denote the image of $\lambda^{u}$ by the inverse map and $\left\{\lambda_{u}, u \in G^{(0)}\right\}$ is a right Haar system on $G$. Let $\mu$ be a quasi-invariant measure on $G^{(0)}$ for the Haar system $\left\{\lambda^{u}, u \in G^{(0)}\right\}, \nu=\int \lambda^{u} d \mu(u)$ be the induced measure by $\mu$ on $G, \nu^{-1}=\int \lambda_{u} d \mu(u)$ be the inverse of $\nu, \nu^{2}=\int \lambda^{u} \times \lambda_{u} d \mu(u)$ be the induced measure by $\mu$ on $G^{(2)}$ and $\Delta$ the modular function of $\mu$. There is a decomposition of the left Haar system $\left\{\lambda^{u}, u \in G^{(0)}\right\}$ for $G$ over $R$. Firstly, there is a measure $\beta_{v}^{u}$ concentrated on $G_{v}^{u}$ for all $(u, v) \in R$ such that $\beta_{u}^{u}$ is a left Haar measure on $G(u)$, and $\beta_{v}^{u}$ is a translate of $\beta_{v}^{v}$ i.e. $\beta_{v}^{u}=x \beta_{v}^{v}$ if $x \in G_{v}^{u}$.

Notice that $\beta_{v}^{u}$ is independent of the choice of $x \in G_{v}^{u}$. Then, there is a unique Borel Haar system $\alpha=\left\{\alpha^{u}\right.$ : 
$\left.u \in G^{(0)}\right\}$ for $R$ with the property that for every $u \in G^{(0)}$, we have $\lambda^{u}=\int \beta_{v}^{\omega} d \alpha^{u}(\omega, v)$. $\mathcal{C}_{c}(G)$ will denote the space of complex-valued continuous functions on $G$ with compact support, endowed with the inductive limit topology and $L^{1}(G, \nu)$ the space of $\nu$ - integrable functions on $G$. In [12], P. Hahn defines the following norm on $L^{1}(G, \nu):\|f\|_{I}=\max \left(\|f\|_{I, r} ;\|f\|_{I, d}\right)$ where $\|f\|_{I, r}=\sup \left\{\int_{G^{u}}|f(x)| d \lambda^{u}(x), u \in G^{(0)}\right\}$, $\|f\|_{I, d}=\sup \left\{\int_{G_{u}}|f(x)| d \lambda_{u}(x), u \in G^{(0)}\right\}$ and introduce the following groupoid algebra,

$$
I(G, \lambda, \mu)=\left\{f \in L^{1}(G, \nu):\|f\|_{I}<\infty\right\}
$$

Under the convolution product defined by: for all $f, g \in I(G, \lambda, \mu)$,

$$
f * g(x)=\int_{G^{r(x)}} f(y) g\left(y^{-1} x\right) d \lambda^{r(x)}(y) .
$$

and the involution defined by: for $f \in I(G, \lambda, \mu)$,

$$
f^{*}(x)=\Delta\left(x^{-1}\right) \overline{f\left(x^{-1}\right)}=\Delta\left(x^{-1}\right) \bar{f}(x) .
$$

$I(G, \lambda, \mu)$ is a Banach *-algebra. Let $K$ be a proper subgroupoid of $G$ with unit space $G^{(0)}$ and equipped with a Haar system $\left\{\gamma^{u}, u \in G^{(0)}\right\}$. As it is explain above, $\left\{\gamma^{u}, u \in G^{(0)}\right\}$ has a decomposition $\left\{\left(\gamma_{v}^{u}\right)_{(u, v) \in R_{K}},\left(\rho^{u}\right)_{u \in G^{(0)}}\right\}$, where $R_{K}$ is the graph of the equivalence relation on $G^{(0)}$ seen as unit space of $K$, such that $\gamma^{u}=\int \gamma_{v}^{\omega} d \rho^{u}(\omega, v)$. We put

$$
I(G \backslash \backslash K)=\left\{f \in I(G, \lambda, \mu): f\left(k x k^{\prime}\right)=f(x) \forall x \in G, \forall k \in K_{r(x)}, \forall k^{\prime} \in K^{d(x)}\right\}
$$

the space of bi- $K$ - invariant integrable functions which is a Banach *-subalgebra of $I(G, \lambda, \mu)$. For any $f \in I(G, \lambda, \mu)$, let us denote by $f^{\natural}$ the bi- $K$-invariant function defined by: for all $x \in G$,

$$
f^{\natural}(x)=\iint f\left(k x k^{\prime}\right) d \gamma_{r(x)}(k) d \gamma^{d(x)}\left(k^{\prime}\right) .
$$

If $I(G \backslash \backslash K)$ is commutative for convolution product, we say that $(G, K)$ is a Gelfand pair. This notion in groupoids case has been studied by authors in $[21,22]$. Let $\mathcal{H}=\left(H_{u}\right)_{u \in G^{(0)}}$ be a Hilbert bundle over $G^{(0)}$ and $\mathcal{U}(\mathcal{H})$ the unitary groupoid of the bundle $\mathcal{H} .(\pi, \mathcal{H})$ is a unitary continuous representation of $\mathrm{G}$ if $\pi$ is a groupoid morphism of $\mathrm{G}$ into $\mathcal{U}(\mathcal{H})$ such that for all square integrable sections $\xi$ and $\eta$ of $\mathcal{H}$, the map $x \mapsto<\pi(x) \xi(d(x)), \eta(r(x))>$ is continuous. A closed nonzero subbundle $\mathcal{M}$ of $\mathcal{H}$ (i.e. $M_{u}$ is a nonzero closed subspace of $H_{u}$ for each $\left.u \in G^{(0)}\right)$ is invariant under $\pi$ if $\pi(x) M_{d(x)} \subset M_{r(x)}$, for each $x \in G$. If $\pi$ admits a non trivial closed invariant subbundle $\mathcal{M}$, it is called reducible. Otherwise it is called irreducible. If $\xi$ is a section of $\mathcal{H}$, the subbundle $\mathcal{M}_{\xi}$ whose leaf at $u \in G^{(0)}$ is the closed linear span of the set $\left\{\pi(x) \xi(d(x)): x \in G^{u}\right\}$ is called the cyclic subbundle generated by $\xi$. We say that $\xi$ is cyclic if $\left(\mathcal{M}_{\xi}\right)_{u}$ is dense in $H_{u}$, for each $u \in G^{(0)}$. We denote by $\Gamma_{\mu}(\mathcal{H})$, the Hilbert space of square integrable section of $\mathcal{H}$. In [20], J. Renault associates to any unitary representation $(\pi, \mathcal{H})$ a representation $L$ of $\mathcal{C}_{c}(G)$ on $\Gamma_{\mu}(\mathcal{H})$ defined by:

$$
(L(f) \xi, \eta)=\int f(x)<\pi(x) \xi(d(x)), \eta(r(x))>d \nu_{0}(x),
$$


for all $f \in \mathcal{C}_{c}(G), \xi, \eta \in \Gamma_{\mu}(\mathcal{H})$, where $\nu_{0}=\Delta^{\frac{-1}{2}} \nu . \quad L$ is a bounded non-degenerate *-representation of $\mathcal{C}_{c}(G)$ where $\mathcal{C}_{c}(G)$ is equipped with the norm $\|\cdot\|_{I}$. We may also define $L$ by: $L(f) \xi(u)=$ $\int_{G^{u}} f(x) \pi(x) \xi(d(x)) \Delta^{\frac{-1}{2}}(x) d \lambda^{u}(x)$. In [18], the authors extend the notion of positive definite function to groupoids. In fact, a bounded continuous function $p: G \rightarrow \mathbb{C}$ is positive definite if for each $u \in G^{(0)}$ and for each $f \in \mathcal{C}_{c}(G)$ we have

$$
\iint f(x) \bar{f}(y) p\left(y^{-1} x\right) d \lambda^{u}(x) d \lambda^{u}(y) \geq 0
$$

Ramsay and Walter establish for groupoids the well-known correspondence between positive definite functions and representations. In fact, for any bounded continuous positive definite function $p: G \rightarrow \mathbb{C}$, there exists a unitary representation $\pi$ of $G$ on a Hilbert bundle $\mathcal{H}$, and a bounded continuous cyclic section $\xi$ of $\mathcal{H}$ such that for each $x \in G, p(x)=<\pi(x) \xi(d(x)), \xi(r(x))>$.

\section{Internally Gelfand Pairs}

Let $G$ be a locally compact, Hausdorff and second countable groupoid and $K$ a proper subgroupoid of $G$.

Definition 3.1. $(G, K)$ is an internally Gelfand pair if for any $u \in G^{(0)},(G(u), K(u))$ is a Gelfand pair.

The first example is given by $\left(G, G^{(0)}\right)$, where $G$ is an abelian groupoid with unit space $G^{(0)}$ which is seen here as a cotrivial groupoid. Let us notice that a groupoid $G$ is said abelian if for any $u \in G^{(0)}$ the isotropy group $G(u)$ is abelian.

The following results give some necessary conditions for internally Gelfand pairs.

Theorem 3.1. Let $L$ be a locally compact group acting continuously on a compact space $S$ and let $T$ be a compact subgroup of $L$ acting trivially on $S$. If $(L, T)$ is a Gelfand pair then the pair of transformation groupoids $(L \propto S, T \propto S)$ is an internally Gelfand pair.

Proof. We have $(T \propto S)(u)=T \subset(L \propto S)(u) \subset L$. Since $(L, T)$ is a Gelfand pair, then $((L \propto S)(u),(T \propto$ $S)(u)$ ) is a Gelfand pair. (see [1])

Consider the relation on $G$ defined by $x \sim y$ iff $r(x)=r(y)$ and $y^{-1} x \in K$. It is an equivalence relation and the quotient space $G / K$, equipped with the quotient topology, is Hausdorff and locally compact. The range map $r$ induces a continuous, open surjection $p: \dot{g} \mapsto r(g)$ from $G / K$ to $G^{(0)}$ (see [20]). The groupoid $G$ acts on $G / K$, that is, the map $(g, s) \mapsto g . s$ from $G * G / K:=\{(g, s) \in G \times G / K: d(g)=p(s)\}$ to $G / K$, is continuous and satisfy
(a) $p(g . s)=r(g)$
(b) $\left(g_{1} g_{2}\right) . s=g_{1} \cdot\left(g_{2} . s\right)$ if $\left(g_{1}, g_{2}\right) \in G^{(2)}$ and 
(c) $p(s) . s=s$ for all $s \in G / K$.

For action groupoids, see [14]. Let us put

$$
G / K \times{ }_{p} G / K=\left\{\left(\dot{g_{1}}, \dot{g_{2}}\right) \in G / K \times G / K: p\left(\dot{g_{1}}\right)=p\left(\dot{g_{2}}\right)\right\}
$$

There is an action of $G$ on $G / K \times{ }_{p} G / K$ defined by the relation: $g .(s, t)=($ g.s,g.t) for $g \in G$ and $s, t \in$ $G / K$.

Theorem 3.2. If for any $s, t \in G / K,(s, t) \sim(t, s)$ then $(G, K)$ is an internally Gelfand pair.

Proof. Let $u \in G^{(0)}$. For $s=u K$ and $x \in G(u)$ we have

$$
\left(s, x^{-1} . s\right)=x^{-1}(x . s, s) \sim(x . s, s) \sim(s, x . s)
$$

Thus there exists $y \in G_{u}$ such that $y . s=s$ and $y x^{-1} . s=x . s$. The first relation shows that $y \in K(u)$ and the second one implies that $x^{-1} y x^{-1} . s=s$. So we have $t \in K(u)$ and $x^{-1} y x^{-1} \in K(u)$. It follows that $x^{-1} \in K(u) x K(u)$ and consequently $(G(u), K(u))$ is a Gelfand pair thanks to Proposition I.2 in [8]. .

Theorem 3.3. Let $G$ be a locally compact, Hausdorff and second countable groupoid and $K$ a proper subgroupoid of $G$. If $(G, K)$ is a Gelfand pair then $(G, K)$ is an internally Gelfand pair.

Proof. Suppose that $(G, K)$ is a Gelfand pair. We consider the map $\psi$ from $L^{1}(G(u) \backslash \backslash K(u))$ to $I(G \backslash \backslash K)$ defined by $f \mapsto F$ where

$F(x)=\int f\left(k x k^{\prime}\right) d \gamma_{r(x)}^{u}(k) d \gamma_{u}^{d(x)}\left(k^{\prime}\right)$ if $K_{u}^{d(x)} \neq \emptyset$ and $K_{r(x)}^{u} \neq \emptyset$ and $F(x)=0$ in other case . Let's show that $F$ is actually in $I(G \backslash \backslash K)$. For $l \in K_{r(x)}, l^{\prime} \in K^{d(x)}$, we assume first that $K_{u}^{d\left(l^{\prime}\right)} \neq \emptyset$ and $K_{r(l)}^{u} \neq \emptyset$. If $k \in K_{u}^{d\left(l^{\prime}\right)}$ then $d(k)=u, r(k)=d\left(l^{\prime}\right)$. Thus $l^{\prime} k$ is defined and $l^{\prime} k \in K_{u}^{r\left(l^{\prime}\right)}=K_{u}^{d(x)}$ that is $K_{u}^{d(x)} \neq \emptyset$. We show in the same way that if $K_{r(l)}^{u} \neq \emptyset$ then $K_{r(x)}^{u} \neq \emptyset$. So,

$$
F\left(l x l^{\prime}\right)=\int f\left(k l x l^{\prime} k^{\prime}\right) d \gamma_{r(l)}^{u}(k) d \gamma_{u}^{d\left(l^{\prime}\right)}\left(k^{\prime}\right)=\int f\left(k x l k^{\prime}\right) d \gamma_{d(l)}^{u}(k) d \gamma_{u}^{r\left(l^{\prime}\right)}\left(k^{\prime}\right)=F(x)
$$

Now, if we assume that $K_{u}^{d\left(l^{\prime}\right)}=\emptyset$ or $K_{r(l)}^{u}=\emptyset$ then $F\left(l x l^{\prime}\right)=0$. If there exists $k \in K_{u}^{d(x)}$ then $r(k)=$ $d(x)=r\left(k^{\prime}\right)$ and $l^{\prime-1} k$ is defined. We have $l^{-1} k \in K_{u}^{d\left(l^{\prime}\right)}$. So, $K_{u}^{d(x)}=\emptyset$ and $F(x)=0$. 
$\psi$ is clearly linear. Now for $f, h \in L^{1}(G(u) \backslash \backslash K(u))$, we set $F=\psi(f)$ and $H=\psi(h)$, we have

$$
\begin{aligned}
F * H(x) & =\int F(y) H\left(y^{-1} x\right) d \lambda^{r(x)}(y) \\
& =\int f\left(k y k^{\prime}\right) h\left(l y^{-1} x l^{\prime}\right) d \gamma_{r(y)}^{u}(k) d \gamma_{u}^{d(y)}\left(k^{\prime}\right) d \gamma_{d(y)}^{u}(l) d \gamma_{u}^{d(x)}\left(l^{\prime}\right) d \lambda^{r(x)}(y) \\
& =\int f\left(k y k^{\prime}\right) h\left(l y^{-1} x l^{\prime}\right) d \gamma_{r(x)}^{u}(k) d \gamma_{u}^{d(y)}\left(k^{\prime}\right) d \gamma_{d(y)}^{u}(l) d \gamma_{u}^{d(x)}\left(l^{\prime}\right) d \lambda_{v}^{d(k)}(y) d \mu(v) \\
& =\int f\left(y k^{\prime}\right) h\left(l y^{-1} k x l^{\prime}\right) d \gamma_{r(x)}^{u}(k) d \gamma_{u}^{d(y)}\left(k^{\prime}\right) d \gamma_{d(y)}^{u}(l) d \gamma_{u}^{d(x)}\left(l^{\prime}\right) d \lambda_{v}^{r(k)}(y) d \mu(v) \\
& =\int f\left(y k^{\prime}\right) h\left(l y^{-1} k x l^{\prime}\right) d \gamma_{r(x)}^{u}(k) d \gamma_{u}^{v}\left(k^{\prime}\right) d \gamma_{v}^{u}(l) d \gamma_{u}^{d(x)}\left(l^{\prime}\right) d \lambda_{r\left(k^{\prime}\right)}^{u}(y) d \mu(v) \\
& =\int f(y) h\left(l k^{\prime} y^{-1} k x l^{\prime}\right) d \gamma_{r(x)}^{u}(k) d \gamma_{u}^{v}\left(k^{\prime}\right) d \gamma_{v}^{u}(l) d \gamma_{u}^{d(x)}\left(l^{\prime}\right) d \lambda_{d\left(k^{\prime}\right)}^{u}(y) d \mu(v) \\
& =\int f(y) h\left(y^{-1} k x l^{\prime}\right) d \gamma_{r(x)}^{u}(k) d \gamma_{u}^{v}\left(k^{\prime}\right) d \gamma_{v}^{u}(l) d \gamma_{u}^{d(x)}\left(l^{\prime}\right) d \lambda_{u}^{u}(y) d \mu(v) \\
& =\int f(y) h\left(y^{-1} k x l^{\prime}\right) d \gamma_{r(x)}^{u}(k) d \gamma_{u}^{d(x)}\left(l^{\prime}\right) d \lambda_{u}^{u}(y) \\
& =\int f * h\left(k x l^{\prime}\right) d \gamma_{r(x)}^{u}(k) d \gamma_{u}^{d(x)}\left(l^{\prime}\right)=\psi(f * h)
\end{aligned}
$$

where the line 7 is due to $K(u)$-biinvariance of $h$.

Thus $\psi$ is a morphism of convolution algebras. Moreover $\psi$ is injective. In fact, let us notice first that if $x \in G(u)$ then $F(x)=\int f\left(k x k^{\prime}\right) d \gamma_{u}^{u}(k) d \gamma_{u}^{u}\left(k^{\prime}\right)=f(x)$. Thus $\psi(f)=\psi(h)$ implies that $F=H$ and in particular, $F_{\mid G(u)}=H_{\mid G(u)}$ that is $f=h$.

The converse is not generally true. For instance if $G$ is abelian, the pair $\left(G, G^{0}\right)$ is an internally Gelfand pair but not a Gelfand pair. For Transitive groupoids, the converse is true (see [21]). In the following result, we give a condition for the converse.

Theorem 3.4. Let $G$ be a locally compact Hausdorf groupoid with unit space $G^{(0)}$ and let $K$ be a proper subgroupoid of $G$ with unit space $G^{(0)}$ such that $(G, K)$ is an internally Gelfand pair. If there exists a unit $u \in G^{(0)}$ such that its orbit in $K,[u]_{K}$, is dense in $G^{(0)}$ then $(G, K)$ is a Gelfand pair.

Proof. Since $(G, K)$ is an internally Gelfand pair then $(G(u), K(u))$ is a Gelfand pair. It follows thanks to

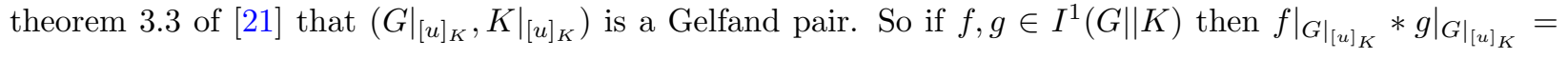

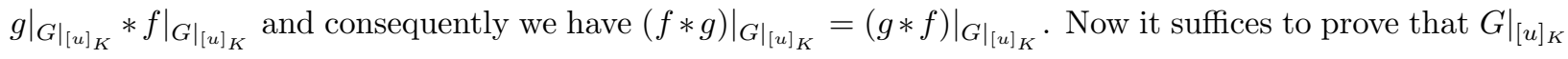
is dense in $G$ to have $f * g=g * f$. In fact, since $[u]_{K}$ is dense in $G^{(0)}$, for $x \in G$ there exists a sequence $\left\{u_{n}\right\}_{n \in \mathbb{N}}$ of elements of $[u]_{K}$ which converges to $d(x)$ in $G^{(0)}$. The map $d$ is continuous surjective and open, so thanks to ( [11], proposition I.25, page 20) there exists a subsequence $\left\{u_{n_{j}}\right\}_{j \in J}$ of $\left\{u_{n}\right\}_{n \in \mathbb{N}}$ and a sequence $\left\{x_{j}\right\}$ of $G$ such that $d\left(x_{j}\right)=u_{n_{j}}$ for any $j \in J$. Since an orbit is an invariant subset of $G^{(0)}$ and $d\left(x_{j}\right) \in[u]_{K}$ then $r\left(x_{j}\right)$ are in $[u]_{K}$. We conclude that $\left\{x_{j}\right\}$ is a sequence of elements of $\left.G\right|_{[u]_{K}}$ converging to $x \in G$. 
Let $\mathcal{H}=\left(H_{u}\right)_{u \in G^{(0)}}$ be a continuous Hilbert bundle over $G^{(0)} . \pi$ is an internally irreducible representation on $\mathcal{H}$ if the restriction of $\pi$ to $G(u)$ is irreducible for any $u \in G^{(0)}$. We denote, as in [3], by $I R e p^{i}(G)$ the set of equivalence classes of internally irreducible unitary continuous representations of $G$ and by $\widehat{G(u)}$ the set of equivalence classes of irreducible unitary continuous representations of $G(u)$. The map $\operatorname{Res}^{u}: I R e p^{i}(G) \mapsto$ $\widehat{G(u)}$, designates the restriction map. Let us denote by $H_{u}^{K(u)}$ the subspace of $K(u)$-invariant vectors defined by $H_{u}^{K(u)}=\left\{h \in H_{u}: \pi(k) h=h, \forall k \in K(u)\right\}$.

Theorem 3.5. If $(G, K)$ is an internally Gelfand pair then for any internally irreducible unitary representation $\pi$ on $\mathcal{H}, \operatorname{dim} H_{u}^{K(u)} \leq 1$ for all $u \in G^{(0)}$.

If for any $u \in G^{(0)}$, Res ${ }^{u}$ is surjective then the converse holds.

Proof. Since $(G(u), K(u))$ is a Gelfand pair and $\pi \mid G(u)$ is irreducible then by classical properties of Gelfand pairs $\operatorname{dim} H_{u}^{K(u)} \leq 1$. For the converse, if $\pi_{u}$ is a unitary irreducible representation of $G(u)$ then, since Res ${ }^{u}$ is surjective, there exists an internally unitary irreducible representation $\pi$ of $G$ such that $\pi_{u}=\pi \mid G(u)$. So $H_{u}^{K(u)}$ is the space of $K(u)$ - invariant vector corresponding to $\pi_{u}$ and it follows that $(G(u), K(u))$ is a Gelfand pair.

Each $H_{u}^{K(u)}$ is a closed subspace of $H_{u}$ so $\mathcal{H}^{K}=\left(H_{u}^{K(u)}\right)_{u \in G^{(0)}}$ is a continuous Hilbert subbundle of $\mathcal{H}=\left(H_{u}\right)_{u \in G^{(0)}}$. We set $A=\left\{u \in G^{(0)}: H_{u}^{K(u)} \neq\{0\}\right\}$

Remark 3.1. The set $A$ is an invariant open subset of $G$.

In fact, let us suppose that for $x \in G, H_{d(x)}^{K(d(x))} \neq\{0\}$. If $\xi_{d(x)}$ is a nonzero vector of $H_{d(x)}^{K(d(x))}$ we set $\eta_{r(x)}=\int \pi(k) \xi_{d(x)} d \gamma_{d(x)}^{r(x)}(k)$. We have

$$
\begin{aligned}
\left\|\eta_{r(x)}\right\| & =\iint<\pi(k) \xi_{d(x)}, \pi\left(k^{\prime}\right) \xi_{d(x)}>d \gamma_{d(x)}^{r(x)}(k) d \gamma_{d(x)}^{r(x)}\left(k^{\prime}\right) \\
& =\iint<\pi\left(k^{\prime-1} k\right) \xi_{d(x)}, \xi_{d(x)}>d \gamma_{d(x)}^{r(x)}(k) d \gamma_{d(x)}^{r(x)}\left(k^{\prime}\right) \\
& =\iint<\xi_{d(x)}, \xi_{d(x)}>d \gamma_{d(x)}^{r(x)}(k) d \gamma_{d(x)}^{r(x)}\left(k^{\prime}\right) \\
& =<\xi_{d(x)}, \xi_{d(x)}>=\left\|\xi_{d(x)}\right\|
\end{aligned}
$$

and for $k_{0} \in K_{r(x)}^{r(x)}$

$$
\pi\left(k_{0}\right) \eta_{r(x)}=\int \pi\left(k_{0} k\right) \xi_{d(x)} d \gamma_{d(x)}^{r(x)}(k)=\int \pi(k) \xi_{d(x)} d \gamma_{d(x)}^{r(x)}(k)=\eta_{r(x)}
$$

Thus $\eta_{r(x)} \in H_{r(x)}^{K(r(x))}$ and is nonzero. So $H_{r(x)}^{K(r(x))} \neq\{0\}$. Now $A$ is open as the support of a continuous field of Hilbert space.

We end this section with some examples 
(1) $G=A \propto S$ a transformation groupoid where $A$ is a locally compact abelian group and $S$ a topological space. Let $L$ be a subgroup of $A$ acting continuously and properly on $S$, so $(G=A \propto S, K=L \propto S)$ is an internally Gelfand pair.

(2) $P(M, G, \pi)$ a principal fiber. $K$ a compact subgroup of $G$. $(G, K)$ is a Gelfand pair if and only if $\left(\frac{P \times P}{G}, \frac{P \times P}{K}\right)$ is an internally Gelfand pair. In fact, $\left(\frac{P \times P}{G}\right)(m)=G$ and $\left(\frac{P \times P}{K}\right)(m)=K$.

(3) Let $G$ be a proper groupoid with unit space $G^{(0)}$. Let us consider a groupoid

$$
\tilde{G}=G \times{ }_{(r, d)} G=\{(x, y) \in G \times G:(r, d)(x)=(r, d)(y)\}
$$

with groupoid structure defined in the following way: $d(x, y)=d(x)=d(y), r(x, y)=r(x)=$ $r(y) ;(x, y)\left(x^{\prime}, y^{\prime}\right)=\left(x x^{\prime}, y y^{\prime}\right)$ if $d(y)=r\left(x^{\prime}\right)$ and $(x, y)^{-1}=\left(x^{-1}, y^{-1}\right)$. The set $\tilde{K}=\{(x, x)$ : $x \in G\}$ is a closed subgroupoid of $\tilde{G}$. We have $\tilde{G}(u)=G(u) \times G(u)$ the cartesian product of $G(u)$ by $G(u)$ and $\tilde{K}(u)=\operatorname{Diag}(G(u) \times G(u))=\{(x, x): x \in G(u)\}$. We know (see [7]) that $(G(u) \times G(u), \operatorname{Diag}(G(u) \times G(u)))$ is a Gelfand pair. So, the pair $(\tilde{G}, \tilde{K})$ is an internally Gelfand pair.

\section{Harmonic Analysis on pairs $(G, K)$}

In this section, $(G, K)$ is an internally Gelfand pair.

Definition 4.1. Let $\varphi$ be a bi-K-invariant continuous function on $G . \varphi$ is $G^{(0)}$-spherical if for any $u \in G^{(0)}$, $\varphi_{\mid G(u)}$, the restriction of $\varphi$ to $G(u)$ is a $K(u)$ - spherical function.

Let's set that $\left.\varphi\right|_{G(u)}=\varphi_{u}$ and $\left.f\right|_{G(u)}=f_{u}$.

Theorem 4.1. Let $\varphi$ be a bi-K-invariant continuous function on $G$ such that $\varphi_{u} \neq 0$ for all $u \in G^{(0)}$. Then $\varphi$ is $G^{(0)}$-spherical if and only if for all $x, y \in G$,

$$
\int \varphi(x k y) d \gamma_{r(y)}^{d(x)}(k)=\varphi(x) \varphi(y)
$$

Proof. Let $\varphi$ be $G^{(0)}$-spherical. Then for any $u \in G^{(0)}$ and $s, z \in G(u)$ we have

$$
\int \varphi_{u}(s k z) d \gamma_{u}^{u}(k)=\varphi(s) \varphi(z)
$$


Now for $x, y \in G$

$$
\begin{aligned}
\int \varphi(x k y) d \gamma_{r(y)}^{d(x)}(k) & =\int \varphi\left((t x l)\left(l^{-1} k k_{1}\right)\left(k_{1}^{-1} y l_{1}\right)\right) d \gamma_{r(x)}^{v}(t) d \gamma_{v}^{d(x)}(l) d \gamma_{v}^{r(y)}\left(k_{1}\right) d \gamma_{v}^{d(y)}\left(l_{1}\right) d \gamma_{r(y)}^{d(x)}(k) \\
& =\int \varphi(t x l)\left(l^{-1} k k_{1}\right)\left(k_{1}^{-1} y l_{1}\right) d \gamma_{r(x)}^{v}(t) d \gamma_{v}^{d(x)}(l) d \gamma_{v}^{r(y)}\left(k_{1}\right) d \gamma_{v}^{d(y)}\left(l_{1}\right) d \gamma_{r\left(k_{1}\right)}^{r(l)}(k) \\
& =\int \varphi(t x l) k\left(k_{1}^{-1} y l_{1}\right) d \gamma_{r(x)}^{v}(t) d \gamma_{v}^{d(x)}(l) d \gamma_{v}^{r(y)}\left(k_{1}\right) d \gamma_{v}^{d(y)}\left(l_{1}\right) d \gamma_{d\left(k_{1}\right)}^{d(l)}(k) \\
& =\iint\left(\varphi(t x l) k\left(k_{1}^{-1} y l_{1}\right) d \gamma_{v}^{v}(k)\right) d \gamma_{r(x)}^{v}(t) d \gamma_{v}^{d(x)}(l) d \gamma_{v}^{r(y)}\left(k_{1}\right) d \gamma_{v}^{d(y)}\left(l_{1}\right) \\
& =\int \varphi(t x l) \varphi\left(k_{1}^{-1} y l_{1}\right) d \gamma_{r(x)}^{v}(t) d \gamma_{v}^{d(x)}(l) d \gamma_{v}^{r(y)}\left(k_{1}\right) d \gamma_{v}^{d(y)}\left(l_{1}\right) \\
& =\varphi(x) \varphi(y)
\end{aligned}
$$

For the converse it suffices to write for a fixed $u \in G^{(0)}$ the equality for $x, y \in G(u)$ and apply the Proposition 6.1 .5 of $[5]$.

Theorem 4.2. Let $\varphi$ be a bi-K-invariant continuous function non identically zero on each $G(u) . \varphi$ is $G^{(0)}$-spherical if and only if for all $f \in I(G \backslash \backslash K)$ there exists a continuous map $\chi_{f}$ on $G^{(0)}$ such that for all $u \in G^{(0)}, \varphi_{u} * f_{u}=\chi_{f}(u) \varphi_{u}$. In particular if there exists a dense orbit $[u]_{K}$ in $G^{(0)}$ then $\chi_{f}$ is constant on $G^{(0)}$.

Proof. For any $u \in G^{(0)}, \varphi_{u}$ is a spherical function on $G(u)$, so for all $f \in I(G \backslash \backslash K)$ there exists a complex number $\chi_{f}(u)$ such that $\varphi_{u} * f_{u}=\chi_{f}(u) \varphi_{u}$. Since $\varphi_{u}$ is spherical then $\varphi_{u}(u)=1$ and it follows that $\chi_{f}(u)=$ $\int_{G(u)} f(x) \varphi(x) d \beta_{u}^{u}(x)$. Thus the continuity of $\chi_{f}$ is due to the continuity of the map $u \mapsto \int_{G(u)} f(x) d \beta_{u}^{u}(x)$ for any $f \in C_{c}(G)$. The converse is trivial. Now if $u \sim_{K} v$ then $\chi_{f}(u)=\chi_{f}(v)$. In fact, for $t \in K_{v}^{u}$ let us consider the map $L_{t}$ from $G(v)$ to $G(u)$ defined by $L_{t}(x)=t x t^{-1} . L_{t}$ is a homeomorphism. If $\beta_{v}^{v}$ is the Haar measure on $G(v)$ then it is straightforward to see that the image measure $\beta_{u}^{u}=L_{t}\left(\beta_{v}^{v}\right)$ is a Haar measure on $G(u)$ and equal to $\beta_{u}^{u}$ since $(G(u), K(u))$ being a Gelfand pair, $G(u)$ is unimodular. Thus

$$
\begin{aligned}
\chi_{f}(u) & =\int_{G(u)} f(x) \varphi\left(x^{-1}\right) d \beta_{u}^{u}(x) \\
& =\int_{G(v)} f\left(t x t^{-1}\right) \varphi\left(t x^{-1} t^{-1}\right) d \beta_{v}^{v}(x) \\
& =\int_{G(v)} f(x) \varphi\left(x^{-1}\right) d \beta_{v}^{v}(x)=\chi_{f}(v)
\end{aligned}
$$

So since $[u]_{K}$ is dense in $G^{(0)}$ and $\chi_{f}$ is continuous then there exists $c \in \mathbb{C}$ such that $\chi_{f}(w)=c$ for all $w \in G^{(0)}$.

Theorem 4.3. Let $\pi$ be an internally irreducible unitary representation on $\mathcal{H}$. $\xi$ a continuous $K$-invariant section such that $\|\xi(u)\|=1$ for any $u \in G^{(0)}$. Then the map $\varphi: x \mapsto \varphi(x)=<\pi(x) \xi(d(x)), \xi(r(x))>$ is a positive definite $G^{(0)}$-spherical function. 
The proof is trivial.

Theorem 4.4. Let $\pi$ be a unitary representation on $\mathcal{H}$ admitting a continuous $K$-invariant section on $G^{(0)}$. If $\operatorname{dim} H_{u}^{K(u)}=1$ for all $u \in G^{(0)}$, then $\pi$ is internally irreducible.

Proof. For all $u \in G^{(0)}, \pi \mid G(u)$ is a unitary continuous representation of $G(u)$ on $H_{u}$. Let $\xi$ be a continuous $K$-invariant section for $\pi$ on $G^{0}$. Then for all $u \in G^{(0)} \xi(u)$ is a $K(u)$-invariant vector for $\pi \mid G(u)$ and since $\operatorname{dim} H_{u}^{K(u)}=1$ then, thanks to Lemme 6.2.3. of [5] (or Proposition 2.6 of [8]), $\pi \mid G(u)$ is irreducible.

A positive definite function $\varphi$ is said $G^{(0)}$ - elementary if the unitary continuous representation associated to it is internally irreducible.

Theorem 4.5. Let $\varphi$ be a bi-K-invariant, continuous, positive definite function such that $\varphi(u)=1$ for all $u \in G^{(0)}$. Then $\varphi$ is $G^{(0)}$ - spherical if and only if $\varphi$ is $G^{0}$ - elementary.

Proof. Let's suppose that $\varphi$ is $G^{(0)}-$ spherical and let $\pi_{\varphi}$ be the unitary representation associated to $\varphi$. We have $\varphi(x)=<\pi(x) \xi(d(x)), \xi(r(x))>$ where $\xi$ is a continuous $K$-invariant section such that $\|\xi(u)\|=1$ for any $u \in G^{(0)}$. Let's set $\varphi_{u}=\varphi_{\mid G(u)}$, the restriction of $\varphi$ to $G(u) . \varphi_{u}$ is $K(u)$ - invariant, continuous, positive definite function such that $\varphi(u)=1$. Since $\varphi_{u}$ is spherical then the representation associated to it, is irreducible and unitarily equivalent to $\left.\pi_{\varphi}\right|_{G(u)}$. So $\pi_{\varphi}$ is internally irreducible. Conversely, $\varphi$ is $G^{(0)}-$ elementary implies that the associated representation is internally irreducible. Thus, the positive definite function $\phi$ associated to $\left.\pi\right|_{G(u)}$ is spherical. But $\phi=\varphi_{u}=\varphi_{\mid G(u)}$. So $\varphi$ is $G^{(0)}-$ spherical.

Denote by $P_{G^{(0)}}$ the set of positive definite $G^{(0)}$ - spherical functions on $G$ and $P_{u}$ the set of positive definite spherical functions on $G(u)$. We know by classical theory (see $[5,8]$ ) that $P_{u}$ equipped with the topology $\sigma\left(L^{\infty}, L^{1}\right)$ is locally compact. For $u \in G^{(0)}$, let's consider $\operatorname{Res}_{u}: P_{G^{(0)}} \rightarrow P_{u}$ the restriction map. If we equip $P_{G^{(0)}}$ with the coarsest topology making continuous the map Res $s_{u}$, then it is locally compact. In this section, we shall suppose that $R e s_{u}$ is bijective. The choice of the topology of $P_{G^{(0)}}$ makes $R e s_{u}$ a continuous open bijection and therefore an homeomorphism. We start by given a definition of the Fourier transform appropriated to our context.

Definition 4.2. For a function $f \in I(G \backslash \backslash K)$, the Fourier transform, noted by $\mathcal{F}(f)$, is defined by: $\mathcal{F}(f)(\varphi)=\int_{G^{(0)}} \int_{G(u)} f(x) \varphi\left(x^{-1}\right) d \beta_{u}^{u}(x) d \mu(u)$ for all $\varphi \in P_{G^{(0)}}$.

We have the following results known in classical case.

Theorem 4.6. (i) For $f, g \in I(G \backslash \backslash K), \mathcal{F}(f * g)=\mathcal{F}(f) \mathcal{F}(g)$

(ii) $\mathcal{F}(f)$ is continuous on $P_{G^{(0)}}$ and vanishing at infinity

(iii) The map $f \mapsto \mathcal{F}(f)$ is a linear transformation. 
Proof. (i)For $f, g \in I(G \backslash \backslash K)$

$$
\begin{aligned}
\mathcal{F}(f * g)(\varphi) & =\int(f * g)(x) \varphi\left(x^{-1}\right) d \beta_{u}^{u}(x) d \mu(u) \\
& =\int f(y) g\left(y^{-1} x\right) \varphi\left(x^{-1}\right) d \lambda^{u}(y) d \beta_{u}^{u}(x) d \mu(u) \\
& =\int f(y) g\left(k y^{-1} x\right) \varphi\left(x^{-1}\right) d \gamma_{d(y)}^{u}(k) d \lambda_{m}^{u}(y) d \beta_{u}^{u}(x) d \mu(m) d \mu(u) \\
& =\int f(y k) g\left(y^{-1} x\right) \varphi\left(x^{-1}\right) d \gamma_{m}^{u}(k) d \lambda_{r(k)}^{u}(y) d \beta_{u}^{u}(x) d \mu(m) d \mu(u) \\
& =\int f(y k) g(x) \varphi\left(x^{-1} y^{-1}\right) d \gamma_{m}^{u}(k) d \lambda_{u}^{u}(y) d \beta_{u}^{u}(x) d \mu(m) d \mu(u) \\
& =\int f\left(k^{\prime} y k\right) g(x) \varphi\left(x^{-1} y^{-1}\right) d \gamma_{u}^{m}\left(k^{\prime}\right) d \gamma_{m}^{u}(k) d \lambda_{r(k)}^{d\left(k^{\prime}\right)}(y) d \beta_{u}^{u}(x) d \mu(m) d \mu(u) \\
& =\int f(y) g(x)\left(\int \varphi\left(x^{-1} k y^{-1}\right) d \gamma_{r(y)}^{d(x)}(k)\right) d \gamma_{u}^{m}\left(k^{\prime}\right) d \lambda_{d(k)}^{r\left(k^{\prime}\right)}(y) d \beta_{u}^{u}(x) d \mu(m) d \mu(u) \\
& =\int f(y) g(x) \varphi\left(x^{-1}\right) \varphi\left(y^{-1}\right) d \gamma_{u}^{m}\left(k^{\prime}\right) d \lambda_{d(k)}^{r\left(k^{\prime}\right)}(y) d \beta_{u}^{u}(x) d \mu(m) d \mu(u) \\
& =\mathcal{F}(f) \mathcal{F}(g)
\end{aligned}
$$

(ii) $\mathcal{F}(f)(\varphi)=\int_{G^{(0)}} \phi(u, \varphi) d \mu(u)$ where $\phi(u, \varphi)=\int_{G(u)} f(x) \varphi\left(x^{-1}\right) d \beta_{u}^{u}(x)$. The map $\varphi \mapsto \phi(u, \varphi)$ is continuous as the composition of continuous functions $\varphi \mapsto \varphi \mid G(u)$ and $\mathcal{F}\left(f_{u}\right)$. Then, we have $|\phi(u, \varphi)| \leq$ $\sup _{u \in G^{(0)}}\left(\int_{G(u)}|f(x)| d \beta_{u}^{u}(x)\right) \leq\|f\|_{I}$. So $\mathcal{F}(f)$ is continuous. Since $\mathcal{F}(f)(\varphi)=\int_{G^{(0)}} \mathcal{F}\left(f_{u}\right)\left(\varphi_{u}\right) d \mu(u)$ and $\mathcal{F}\left(f_{u}\right)$ vanishing at infinity then $\mathcal{F}(f)$ is vanishing at infinity.

(iii) The proof is trivial.

Theorem 4.7. Let $\phi$ be a bi-K-invariant continuous positive definite function on $G$ such that $\phi(u) \leq 1$ for all $u \in G^{(0)}$. Then there exists a unique measure $\Gamma$ on $M_{0}\left(P_{G^{(0)}}\right)$ such that for all $x \in G$,

$$
\phi(x)=\int_{P_{G}(0)} \omega(x) d \Gamma(\omega)
$$

Proof. For any $u \in G^{(0)}, \phi_{u}$ the restriction of $\phi$ on $G(u)$ is bi- $K(u)$-invariant continuous positive definite function on $G(u)$. Thus thanks to Bochner theorem for Gelfand pairs, there exists a unique measure $\theta_{u}$ on $M_{0}\left(P_{u}\right)$ such that for all $x \in G^{(0)}, \phi_{u}(x)=\int_{P_{u}} w_{u}(x) d \theta_{u}\left(w_{u}\right)$. We consider then the measure image $\Gamma^{u}$ of $\theta_{u}$ by $\operatorname{Res}_{u}^{-1}$. So we obtain a family of measure $\left\{\Gamma_{u}: u \in G^{(0)}\right\}$ on $P_{G^{(0)}}$. We put $\Gamma=\int_{G^{(0)}} \Gamma^{u} d \mu(u)$. For all $x \in G$ we have

$$
\begin{aligned}
\phi(x) & =\int \phi\left(k x k^{\prime}\right) d \gamma_{r(x)}^{u}(k) d \gamma_{u}^{d(x)}\left(k^{\prime}\right) d \mu(u) \\
& =\int \omega_{u}\left(k x k^{\prime}\right) d \theta_{u}\left(\omega_{u}\right) d \gamma_{r(x)}^{u}(k) d \gamma_{u}^{d(x)}\left(k^{\prime}\right) d \mu(u)
\end{aligned}
$$




$$
\begin{aligned}
& =\int \operatorname{Res}_{u}(\omega)\left(k x k^{\prime}\right) d \Gamma(\omega) d \gamma_{r(x)}^{u}(k) d \gamma_{u}^{d(x)}\left(k^{\prime}\right) d \mu(u) \\
& =\int \omega(x) d \Gamma^{u}(\omega) d \mu(u) \\
& =\int \omega(x) d \Gamma(\omega)
\end{aligned}
$$

\section{REFERENCES}

[1] M. Akouchi, A. Bakali, Une Généralisation des paires de Gel'fand, Bolletino U.M.I, 7 (1992), 795-822.

[2] M. Buneci, Groupoid Algebras For Transitive Groupoids, Math. Rep., 5(55) (2003), 9-26.

[3] R. Bos, Groupoids in Geometric Quantization, PhD. Thesis, Radboud University Nijmegen, 2007.

[4] J. Dieudonné, Gelfand Pairs And Spherical Functions, Int. J. Math. Math. Sci., 2(2) (1979), 153-162.

[5] V. Dijk, Introduction to Harmonic Analysis and Generalized Gelfand Pairs, De Gruyter Stud. Math., 36, Walter de Gruyter And Co, Berlin, 2009.

[6] J. Dixmier, Les $C^{*}$-Algèbres et Leurs Représentations, Gauthier-Villars, Paris, 1964.

[7] J. Faraut, Infinite Dimensional spherical Analysis, COE Lecture Note, 10, Kyushu University, 2008.

[8] J. Faraut, Analyse Harmonique sur les Paires de Gelfand, Les cours du CIMPA, 1980.

[9] I. F. Gelfand, Spherical Functions On Symetrics Spaces, Dokl. Akad. Nauk., 70 (1960), 5-8.

[10] R. Godement, A Theory Of Spherical Functions I, Trans. Amer. Math. Soc., 73 (1952), 496-556.

[11] G. Goehle, Groupoid Crossed Products, PhD. Thesis, Dartmouth College, 2009.

[12] P. Hahn, The regular representations of measure groupoids, Trans. Amer. Math. Soc., 242 (1978), 34-72.

[13] P. Hahn, Haar measure for Groupoids, Trans. Amer. Math. Soc., 242 (1978), 1-33.

[14] K. Mackenzie, General Theory of Lie Groupoids and Lie Algebroids, London Math. Soc. Lecture Note Ser., 213, Cambridge University Press, 2005.

[15] J. F. M_\{c\}Clendon, Metric Families, Pac. J. Math., 57 (1975), 491-509.

[16] P. S. Muhly, J. N. Renault, D. P. Williams, Continuous-Trace Groupoid C*-algebras, III, Trans. Math. Soc., 348 (1996), 3621-3641.

[17] K. J. Oty, Fourier-Stieltjes Algebras of r-Discrete Groupoids, J. Oper. Theory, 4 (1999), 175-197.

[18] A. Ramsay, M. E. Walter, Fourier-Stieltjes Algebras of Locally Compact Groupoid, J. Funct. Anal., 148(2) (1997), $314-367$.

[19] J. Renault, The Ideal Structure of Grupoid Crossed Product $C^{*}$-Algebras, J. Oper. Theory, 25 (1991), 3-36.

[20] J. Renault, A Groupoid Approach to $C^{*}$-Algebras, Lecture Notes in Math., 793, Springer-Verlag, 1980.

[21] I. Toure, K. Kangni, On Gelfand Pairs associated to transitive groupoids, Opuscula Math., 33(4) (2013), 751-762.

[22] I. Toure, K. Kangni, On Groupoid Algebras of Biinvariant Functions, Int. J. Math. Anal., 6 (43) (2012), 2101-2108.

[23] J. J. Westman, Harmonic Analysis on groupoids, Pacific J. Math., 27(3) (1968), 621-632. 